\title{
Implantation of Esterified Hyaluronic Acid in Microdissected Reinke's Space after Vocal Fold Microsurgery: First Clinical Experiences
}

Camille Finck, MD; Philippe Lefebvre, MD, PhD

Objective: In this pilot study are presented the first clinical experiences of the use of a resorbable bioimplant made of esterified hyaluronic acid inserted in the microdissected superficial layer of the lamina propria (SLLP), also called Reinke's space, after a flap excision procedure for a benign vocal fold lesion. Laryngeal and vocal evolution of implanted patients are depicted and discussed. Study Design: Eleven bio-implants have been inserted in microdissected SLLP of 11 cases presenting with benign vocal fold lesions. The surgical procedure consisted of the excision of primary lesion by a microflap technique immediately followed by implantation of esterified hyaluronic acid in Reinke's space. Methods: All patients underwent rigid laryngoscopy and a microsurgical procedure under general anesthesia. The cordal lesion was treated with cold instrumentation of Bouchayer ( 7 cases) or with a mixed technique using $\mathrm{CO}_{2}$ laser (4 cases). After the classical freeing-up of Reinke's space and the creation of a mucosal flap, a few fibers of esterified hyaluronic bioimplant are gently arranged in Reinke's space before redraping the ligament and closing the cordal incision with a few drops of fibrin glue. Laryngeal and vocal assessments were performed pre- and postoperatively in all patients using videostroboscopy as well as perceptual and objective voice evaluation. All patients were followed in a longitudinal manner: between two and five postoperative evaluations were performed. The longest follow-up was 19 months and the shortest 2 months. Results: All cases exhibited postsurgical improvement of the pliability of the SLLP. None of them developed an adverse scarring process. Improvement

From the Department of Otorhinolaryngology, University of Liège, Liège, Belgium. 2005.

Editor's Note: This Manuscript was accepted for publication May 27,

This work was made possible thanks to a grant from University of Liège/CHU (FIRS 4737) and to a research grant from Schering Plough.

Send Correspondence to Dr. Camille Finck, Department of Otorhinolaryngology, CHU Sart Tilman, University of Liège, B-4000 Liège, Belgium. E-mail: camille.finck@hu.ulg.ac.be

DOI: 10.1097/01.mlg.0000173158.22274.8d of SLLP's pliability was maintained in time in all cases. Vocal improvement was observed in all. Temporary inflammation was noted in one case. There were no serious adverse effects apparent during the follow-up period. Conclusion: Bio-implantation of esterified hyaluronic acid in Reinke's space is technically easy and well tolerated. All treated cases exhibited postoperative good pliability of the SLLP compared with their preoperative evaluation. Key Words: Esterified hyaluronic acid, SLLP, Reinke's space, vocal fold microsurgery, vocal fold bio-implantation.

Laryngoscope, 115:1841-1847, 2005

\section{INTRODUCTION}

Normal human vocal folds (VF) have a very specialized and unique laminar histologic architecture. Under the squamous epithelium lie the three layers of connective tissue called the lamina propria (LP). Those layers have a distinct structure and also different mechanical properties. The superficial layer of the lamina propria (SLLP), also called Reinke's space, is located just under the epithelium. More deeply located, the intermediate layer rich in elastic fibers and the deep layer rich in collagen fibers, form together the vocal ligament. Under the LP lies the thyroarytenoid muscle or vocal muscle.

In the body-cover model of vibration proposed by Hirano, ${ }^{1}$ the cover is composed of the epithelium and the SLLP. In this model, the natural pliability of the SLLP is essential to normal voice production. The cellular composition of SLLP is very sparse, made of fibroblasts and macrophages. Its "jelly like" structure is caused by a very loose elastic and collagen fibrous scaffolding and by the interstitium molecules of the extracellular matrix (ECM). Among those molecules, hyaluronic acid or hyaluronan (HA), a glycosaminoglycan, which is a normal constituent of the ECM everywhere in the body, appears to play an important role in determining the biological, mechanical, and wound healing characteristics of the SLLP. ${ }^{2} \mathrm{HA}$ is a high molecular weight glycosaminoglycan first described by Meyer and Palmer ${ }^{3}$ in 1934 who extracted the molecule 
from the vitreous humor of the eye. HA influences the thickness of the LP: men possess a thicker LP compared with women, and this could be caused by a three to fourfold amount of HA in the men's LP. ${ }^{4}$

HA not only influences thickness of SLLP but also its visco-elastic characteristics: HA is necessary to maintain both optimal viscosity and stiffness of SLLP. In 1999, Gray et al. $^{5}$ showed that the removal of HA from the SLLP of two human cadavers increased the tissue viscosity by two to fourfold. Stiffness of the SLLP is also influenced by the amount of HA present, which in turn influences the stability of the frequency of vibration: a less stiff SLLP leads to a more unstable vibratory rate. In 2001, Chan et al. ${ }^{6}$ demonstrated that removal of HA from the LP of five adult male cadavers decreased the stiffness of the VF cover by an average of $35 \%$ and increased dynamic viscosity by $70 \%$.

HA deeply influences cell behavior and appears to be an important regulator of wound repair and of morphogenetic events. Previous studies have shown that HA influences collagen deposition and that high levels of HA reduce scar tissue formation with less fibrosis and less contracture. ${ }^{7}$

The growing knowledge of the natural biomechanical properties of the VF main vibrator, the mucosal cover, has influenced the microsurgical procedures for the treatment of benign VF lesions: restoration of mucosal vibratory function is known to be the most important goal to achieve to improve not only the quality of voice but the aerodynamic effectiveness of the laryngeal vibrator and hence the ease of phonation. Many authors have stressed the importance of preserving as much SLLP as possible during cordal microsurgery. ${ }^{8,9}$ When SLLP is damaged to a sufficient extent, its molecular structure is modified: the normal proteoglycans and glucosaminoglycans such as HA are replaced by collagen fibers and fibronectin 8,10 and scar tissue is created, resulting in increased stiffness of the mucosal cover and reduced mucosal motion during cordal oscillation ${ }^{11}$ because of the change in the viscoelastic properties of the scarred mucosal cover.

In our personal experience, even extremely careful VF microsurgery represents a potential risk of local adhesion of the epithelial basal membrane to the ligament: in case of wide dissection of the Reinke's space (such as in microsurgical treatment of Reinke' s edema), in case of a greater loss of tissue than expected inside the SLLP (such as in microdissection of type III intracordal cysts), ${ }^{8}$ or when the microsurgically treated pathology is primarily characterized by an absence of normal SLLP (such as in submucosal localized fibrosis or like in type II vergeture).

Knowing the favorable and important biological and mechanical roles played by HA in normal SLLP, we have been interested in using an HA-derivative bioimplant in those surgical cases where a deeply damaged SLLP is observed. The implant should be resorbable to play only a temporary role: it should prevent the early adhesion of the epithelial basement membrane to the underlying ligament and if possible allow a better healing process of the SLLP.

Many exogenous HA preparations are commercially available and have been proven to be nontoxic and nonantigenic because of the absence of proteins, as well as perfectly biocompatible. They have been used extensively, for many years, in many surgical specialties such as ophthalmic, gynecologic, abdominopelvic, plastic, and osteoarthritic surgery. ${ }^{12}$ The objectives of the current prospective study are to evaluate the laryngeal function and vocal results obtained after implantation of a modified HA bioimplant, esterified hyaluronic acid (EHA), under the mucosal flap, in the Reinke's space, after a microsurgical procedure.

\section{MATERIALS AND METHODS}

\section{Subjects}

Eleven patients ( 3 men and 8 women) who underwent a microsurgical procedure on their vocal cords were selected for treatment between February 14, 2003 and October 1, 2004. All of them presented with benign lesions of the VFs and underwent a microsurgical procedure and implantation of EHA in Reinke's space. Diagnosis, sex, age, surgical technique (with or without laser), and personal characteristics are depicted in Table I. Two cases benefited from a bilateral procedure, but only one side was implanted.

\section{Procedure}

All patients underwent general anesthesia and routine laryngeal intubation (tracheal tube size: 5.5-6.5). A direct laryngoscopy was performed in all patients (Kleinsasser type laryngoscope, Storz $8590 \mathrm{C}$ and 8590B). The microsurgical procedure was done under $\times 25$ magnification (Microscope Leica M655). The cordal lesion was treated either with cold instrumentation of Bouchayer only (Microfrance) or with CO2 Laser (Sharplan 40C). The surgical procedure accomplished the classical liberation of Reinke's space and the creation of a mucosal flap to redrape the cordal ligament. For the patients with Reinke's edema, after suctioning of the submucosal edema, care was taken not to sacrifice too large an amount of mucosa to be able to close the incision without any superficial defect.

The decision to use a bioimplant made of EHA was taken at the time of the surgical procedure and was linked to an estimated higher surgical damage to the SLLP. A few fibers of EHA bioimplant are taken with the microforceps and gently arranged in Reinke's space between the ligament and the mucosal flap. The cordal mucosal flap is then precisely closed above the implant and maintained in place by using two or three drops of Fibrin glue (Tissucol DUO 500 ${ }^{\circ}$, 0,5 mL Baxter, Deerfield, Il). Surgical photographs of the procedure performed in case 11 suffering of left VF mucous cyst are presented in Figure 1.

Postoperatively, all patients were advised to adhere to a vocal rest for 8 days. Peroperatively, they received a $125 \mathrm{mg}$ intravenous injection of methylprednisolone. Medical postoperative treatment consisted of degressive oral methylprednisolone, clobutinol hydrochloride, paracetamol, and amoxyclavulanate for 1 week.

\section{Bioimplant}

The commercially available EHA presents as a fiber material made of HYAFF (Fidia Advanced Biopolymers, Abano, Terme, Italy). The HYAFF polymers are modified HA derived by esterification of the carboxyl group of the glucuronic acid moiety of the polymer with linear or aromatic alcohols. The EHA implant used in this study is HYAFF 11 (MeroGel), in which $100 \%$ of carboxyl groups of HA are esterified with benzyl alcohol and is a registered trade mark of Medtronic Xomed, Jacksonville, FL.

When placed in contact with the human body fluids, the fiber-like material transforms itself into a highly hydrated gel, 
TABLE I.

Subjects.

\begin{tabular}{|c|c|c|c|c|c|c|c|c|c|}
\hline & & Diagnostic & $\begin{array}{l}\text { Voice } \\
\text { Professional }\end{array}$ & $\begin{array}{c}\text { Smoking } \\
\text { Habit }\end{array}$ & Age & $\begin{array}{c}\text { Unilateral } \\
\text { Microsurgery }\end{array}$ & $\begin{array}{c}\text { Bilateral } \\
\text { Microsurgery }\end{array}$ & Bioimplant & Laser \\
\hline Case 1 & M & Right submucosal fibrosis & Yes & No & 51 & $x$ & & Right & No \\
\hline Case 2 & M & Right intracordal open cyst & No & $\begin{array}{l}\text { Stopped } \\
\text { (in 1994) }\end{array}$ & 74 & $x$ & & Right & No \\
\hline Case 3 & $\mathrm{~F}$ & Right intracordal organized hematoma & No & No & 47 & $\mathrm{X}$ & & Right & No \\
\hline Case 4 & $\mathrm{~F}$ & Bilateral Reinke's edema & No & Yes & 68 & & $x$ & Left & Yes \\
\hline Case 5 & $\mathrm{~F}$ & $\begin{array}{l}\text { Bilateral Reinke's edema, left open cyst, } \\
\text { and localized vergeture }\end{array}$ & No & Yes & 44 & $x$ & & Left & Yes \\
\hline Case 6 & $\mathrm{~F}$ & $\begin{array}{l}\text { Right intracordal open cyst, bilateral } \\
\text { hypotony }\end{array}$ & Yes & No & 27 & $\mathrm{x}$ & & Right & No \\
\hline Case 7 & $\mathrm{~F}$ & Bilateral Reinke's edema & No & Yes & 70 & & $\mathrm{x}$ & Left & Yes \\
\hline Case 8 & $\mathrm{~F}$ & Right Reinke's edema & No & Stopped & 44 & $\mathrm{x}$ & & Right & Yes \\
\hline Case 9 & $\mathrm{~F}$ & Left cordal vergeture & No & No & 35 & $x$ & & Left & No \\
\hline Case 10 & M & Left cordal vergeture, right sulcus & No & No & 12 & $x$ & & Left & No \\
\hline
\end{tabular}

which is then totally degradable through hydrolysis of the ester bond, releasing free alcohol and HA. The two molecules are then metabolized in the body through their normal metabolic degradation pathways. ${ }^{13}$

\section{Laryngoscopic Examination}

All patients underwent a general ear, nose, and throat clinical examination. Videolaryngostroboscopy was performed preoperatively and postoperatively. We used a Wolff rigid $70^{\circ}$ endoscope 4450.47 connected to a Wolff laryngostroboscope 5052. All patients were videotaped on an Olympus camera Visera OTV57.Videotapes of each patient were acquired using a video recorder PanasonicAG-5700. Video printouts of a Sony UP-3000P video printer were conserved for data files. Topical anesthesia of the pharynx (xylocaïne 10\% spray) was used. The patients were asked to sustain an /ê/ at different pitches.

Amplitude of vibration and of mucosal wave as well as
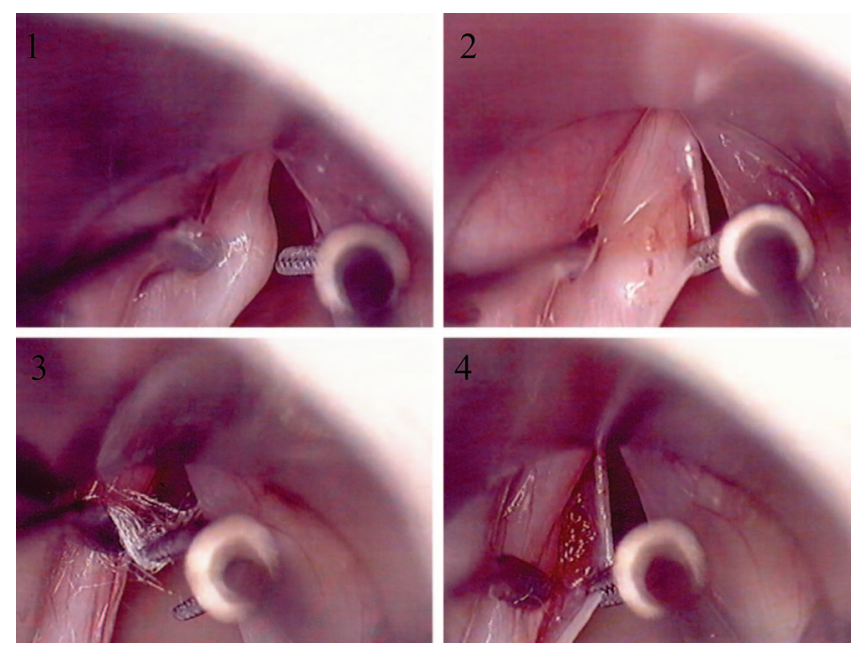

Fig. 1. Surgical photographs of case 11's procedure (left vocal fold [VF] mucous cyst). (1) Preoperative microlaryngoscopic view of left VF mucous cyst. (2) Opening of the Reinke's space. (3) Presentation of MeroGel fibers before insertion in the microdissected superficial layer of the lamina propria. (4) MeroGel implant placed under the mucosal flap. symmetry, inflammation, presence of a glottic gap, and of VF deformation are the videostroboscopic findings that were taken into account. Amplitude of vibration, mucosal wave and glottic gap were evaluated on an analogic scale.

For amplitude vibration and mucosal wave, we used a $10 \mathrm{~cm}$ scale. Amplitude of vibration is considered normal if noted at 5, increased if above 5, and decreased below 5 .

Mucosal wave is considered normal if noted at 10. Every value between 10 and 0 is considered a decreased wave. A wave noted 0 is a completely absent wave.

For glottic closure, a scale of $5 \mathrm{~cm}$ is used. Glottic closure is considered complete at 5 and diminished if noted between 0 and 5 .

\section{Voice Evaluation}

The voices were recorded in a soundproof booth on a Kay Elemetrics Computerized Speech Lab with a Kay Elemetrics head-mounted microphone. The distance between the mouth and the microphone was approximately $3 \mathrm{~cm}$. Three sustained vowels /a/ and five standardized texts for French were recorded at each visit. We used the GRBASI scale for the subjective evaluation of the patient's voice quality, after listening to the five productions of the standardized texts: G(rade), R(oughness), B(reathiness), $\mathrm{A}$ (sthenia), $\mathrm{S}$ (train), I (nstability). Each item is quoted as 0 (normal), 1 (light), 2 (moderate), and 3 (severe).

Software from Kay Elemetrics, Multi-Dimensional Voice Program (MDVP) was used to obtain voice quality objective data. Measurements of average fundamental frequency (average Fo), of highest and lowest fundamental frequency (Fhi, Flo), SD of Fo (STD), phonatory Fo range (PFR), jitter ratio (Jitt), and noise-toharmonic ratio (NHR), were derived from 3 second segments of each of the sustained /a/. For patient 3 , the preoperative objective voice evaluation was obtained from the CSL Main Program Software because intensity of voice was too low to permit the use of MDVP Software.

\section{RESULTS}

All the results obtained can be organized in accordance with the time spent after surgery:

1. Early postoperative evaluation (EPOE): between 1 and 2 weeks after surgery. 
2. Intermediate postoperative evaluation (IPOE): between 3 and 4 weeks after surgery.

3. Late postoperative evaluation (LPOE): between 7 and 12 weeks after surgery.

4. Long-term postoperative evaluation (LTPOE): between 15 and 38 weeks after surgery.

5. Very long-term postoperative evaluation (VLTPOE): available in 3 cases, between 10 and 19 months.

Preoperative values for case 7 are obtained after her first microsurgery on the right VF (cure of a huge Reinke's edema) with no bioimplantation realized on that right VF. No serious adverse effect was noted during the observation period.

\section{Laryngoscopic Examination}

Figure 2 shows the videostroboscopic findings of the 11 cases in three separate graphs.

Inflammation. Only case 7 suffered from a significant inflammation after treatment: the VF appeared deprived of any vibratory movement or mucosal wave at EPOE. Voice rest for 2 weeks and a medical treatment consisting of methylprednisolone and ultrasonic humidifying solution (propylene glycol 5\% in a physiologically balanced salt solution) permitted the patient to regain an almost normal wave at IPOE

Vocal fold general aspect. Concerning the general appearance of the treated VF observed at the EPOE, all of them except case 7 already exhibited a noninflammatory, smooth, well-hydrated, good looking aspect at rest. This was particularly striking in the two worst cases: case 3 suffering from an intracordal organized hematoma and case 10 suffering from an extremely deep and long vergeture.

A slight convexity of the implanted VF was visible at EPOE in case 1,2 , and 10 but rapidly disappeared and was no longer visible at IPOE. Case 3 still exhibited a very slight deformation of the free margin of the treated $\mathrm{VF}$ at LPOE. In case 11, who was suffering from an anterior left mucous cyst, comparison of the general aspect of the treated VF at EPOE and LPOE shows a change in the color of the surgical zone together with a straighter upper lip of the VF.

Amplitude of vibration. Amplitude of vibration increased after bio-implantation in all of the eight cases who had a decreased preoperative amplitude. We lack EPOE for case 4, whereas case 9 exhibited extreme tension and vocal inhibition, which lead to the impossibility to obtain normal vibration and stroboscopic assessment. The 3 cases who experienced a decreasing amplitude after surgery had a preoperative increased vibratory amplitude (cases 5, 7, and 8).

Mucosal wave. Preoperatively, a mucosal wave was absent or decreased in 8 of 11 cases (cases 1, 2, 3, 5, 6, 9, 10,11 ) and normal in 3 of 11 cases (cases 4,7 , and 8 ). The cases with a normal preoperative mucosal wave were those with Reinke's edema. All cases with a decreased wave experienced an increasing mucosal wave after microsurgery and bio-implantation. Case 7 , who suffered from a significant inflammatory response at the early postsurgical check up, with no mucosal wave visible at the time, regained an almost normal wave at the IPOE.
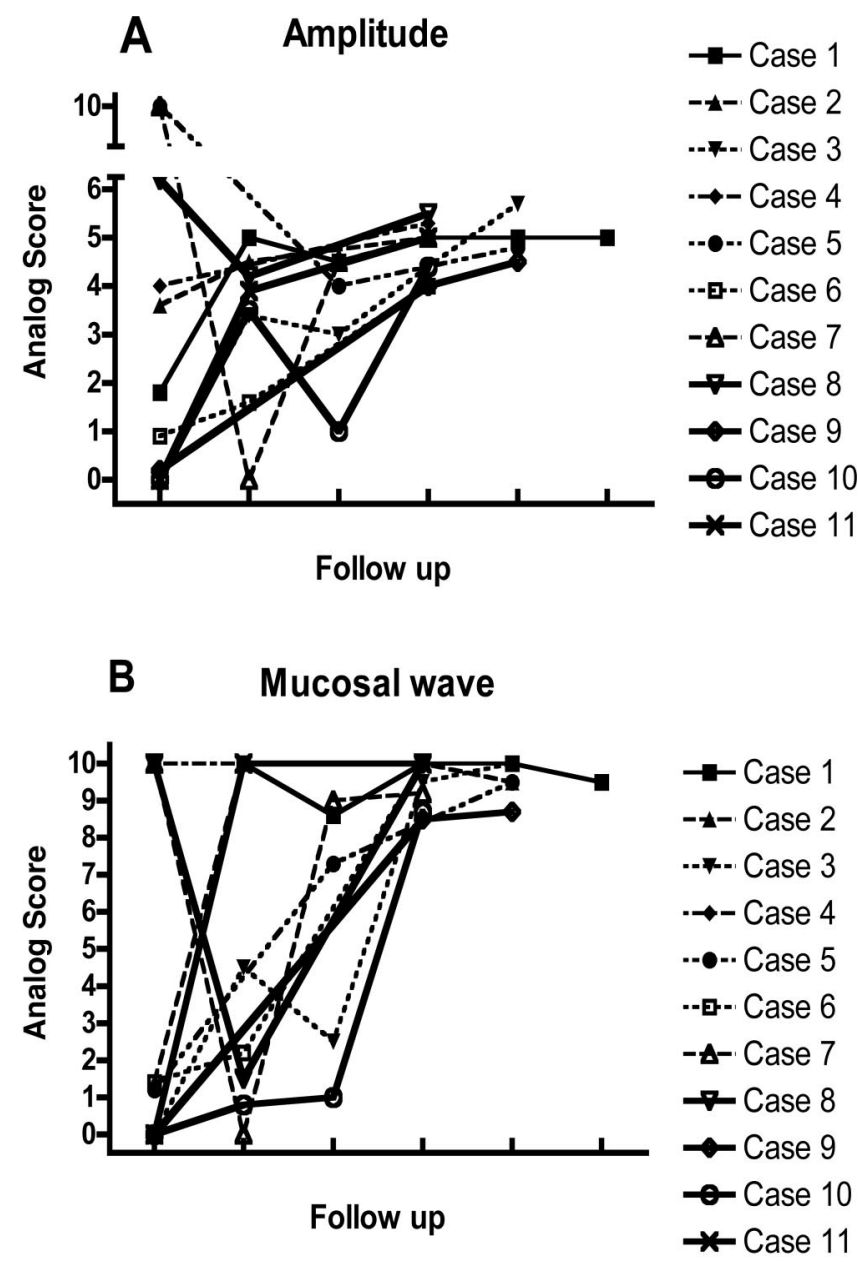

C Glottic closure

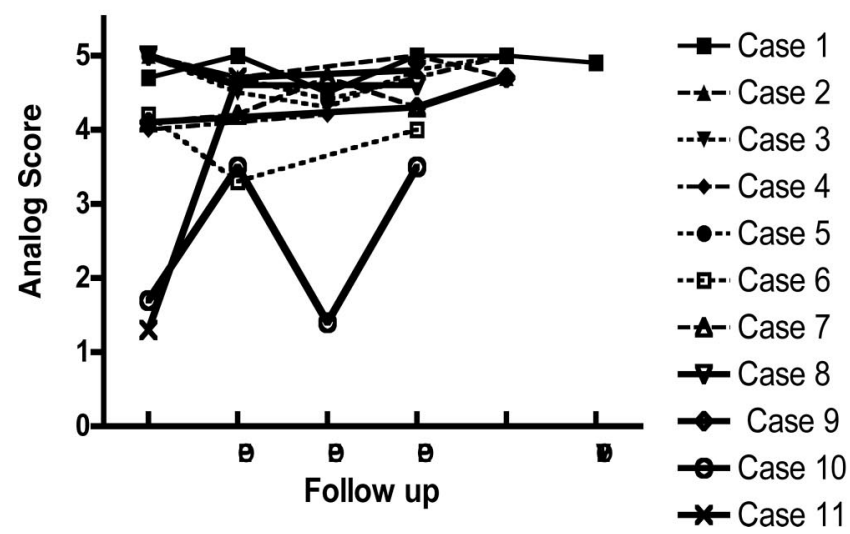

Fig. 2. Videostroboscopic data. (A) Amplitude of vibration. (B) Amplitude of mucosal wave. (C) Glottic closure. PREO = preoperative evaluation; $\mathrm{EPOE}=$ early postoperative evaluation; IPOE = intermediate postoperative evaluation; LPOE = late postoperative evaluation; LTPOE = long-term postoperative evaluation; VLTPOE = very long-term postoperative evaluation.

Glottic closure. Glottic closure improved in 6 of 11 cases (cases 1, 4, 7, 9, 10,11), was unchanged in 2 cases (cases 3 and 5), and was slightly diminished after surgery in 3 cases of 11 (cases $2,6,8$ ). 


\section{Voice Evaluation}

Perceptual voice evaluation. Table II depicts the perceptual voice evaluation of the cases according to the time of surgery.

All our cases experienced a progressive improvement of voice with the passage of time after the microsurgical procedure. A grade 1 dysphonia was achieved by 6 of 11 cases in a period of time ranging from 1 to 12 weeks. The worst result was observed in case 10 and case 5 , who still exhibit a grade 3 dysphonia at LTPOE and LPOE, respectively.

Objective voice quality data. Objective voice quality data are presented in Figure 3. The values are the mean of the values obtained from the three consecutive sustained /a/ measured on the MDVP software.

STD and PFR show a degressive pattern after microsurgery and bio-implantation in 9 cases $/ 11$ cases (cases 1 , $2,3,4,6,7,8,9,11)$. Case 5 is the only patient exhibiting an increase in the value of STD and PFR. Case 10 exhibits very fluctuant values.

Considering LPOE and LTPOE, Jitt diminished in six cases (cases $2,3,7,8,9,11$ ), increased in two cases (cases 5 and 6), and was almost unchanged in two cases (cases 1, 4, and 10).

NHR shows a decreased pattern after microsurgery and bio-implantation in 8 cases of 11 (cases 1, 2, 3, 4, 5, 7, 8,9 ) and an increase in 2 cases (cases 6 and 10) at the LTPOE. NHR is almost unchanged in case 11, after an initial drop of its value. The best result was obtained in patient 3.

\section{DISCUSSION}

The objective of this pilot study is to evaluate the laryngeal function and vocal results obtained after insertion of a resorbable EHA implant under the mucosal flap, in the Reinke's space, at the end of a microsurgical procedure for benign cordal lesions. The decision of using the implant was in all cases guided by the estimated risk of a too important damage to the SLLP, primary or postsurgical, which could preclude favorable postsurgical vibratory evolution. Because of the small size of this series and the inhomogeneous nature of the treated pathologies, evaluation of the results obtained will be performed in a longitudinal perspective.

All the observed cases showed an early excellent general aspect of the treated VF, except case 7, who experienced inflammation. All cases showed an excellent postoperative mucosal wave and vibratory amplitude. In all observed cases, mucosal wave improvement had reached its maximum rating at LPOE or LTPOE, whereas early improvement was already seen in six cases.

Case 4, who underwent bilateral microsurgical treatment for a severe Reinke's edema, exhibited a better amplitude of vibration on the bioimplanted side compared with the side that was not implanted. Unilateral insertion of EHA was realized on one side because one mucosal flap was tighter, and we were concerned with the risk of extrusion of the bioimplanted material on that side.

\section{Bioimplantation is Well Tolerated, the General Aspect of Treated VF Being Excellent; Inflammation has been Observed in Only Case 7}

In this particular case, we believe that the use of the $\mathrm{CO}_{2}$ laser combined with an initially too lateral mucosal incision with exposition of the vocal ligament probably played the major role in the development of a considerable inflammatory response. The appearance of the VF was similar to inflammatory responses observed in our experience after laser cordectomies type I.

\section{Time Evolution of Laryngeal Images Shows Excellent Early but also Long-Term Tolerance of EHA Bioimplant}

The VLTPOE available in three cases $(1,2$, and 4$)$ shows an excellent stability of the laryngeal image with a slight continued increase of the amplitude of movement in case 2 and 4, who are finally rated as having a lateral movement of vibration slightly above the normal amplitude. Case 4 also reached complete glottic closure at that very long-term evaluation. No adverse local reactions such as granuloma or stiffness have been observed in any of our cases.

TABLE II.

Perceptual Voice Evaluation: GRBASI Scale.

\begin{tabular}{|c|c|c|c|c|c|c|}
\hline & Preoperative Evaluation & EPOE & IPOE & LPOE & LTPOE & VLTPOE \\
\hline Case 2 & 220022 & 221010 & - & 110011 & 110011 & 110011 (19 months) \\
\hline Case 4 & 331033 & - & 211103 & 111001 & - & 000000 (10 months) \\
\hline Case 5 & 331013 & - & 111001 & - & 320011 & \\
\hline Case 6 & 231013 & 110102 & - & 111112 & - & \\
\hline Case 8 & 320011 & 211011 & - & 221001 & & \\
\hline Case 9 & 333033 & $3-3-$ & - & 211032 & 111121 & \\
\hline Case 10 & 333033 & 321223 & 222132 & 323233 & & \\
\hline Case 11 & 333033 & 111131 & - & 111101 & & \\
\hline
\end{tabular}

GRABASI = grade, roughness, breathiness, asthenia, strain, instability; EPOE = early postoperative evaluation; IPOE = intermediate postoperative evaluation; LPOE = late postoperative evaluation; LTPOE = long-term postoperative evaluation; VLTPOE = very long-term postoperative evaluation. 

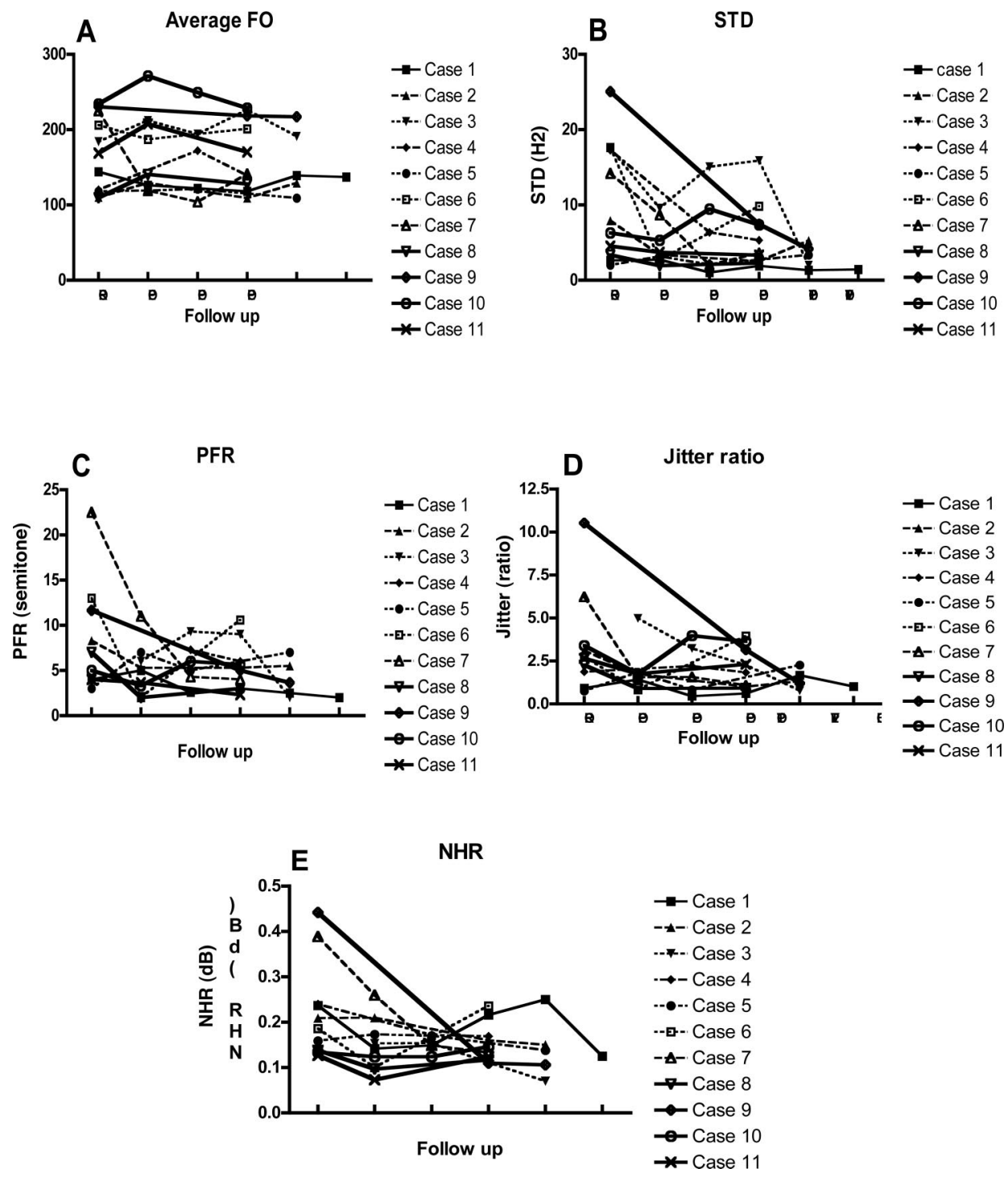

Fig. 3. Objective voice data. (A) Average fundamental frequency (Fo). (B) Standard deviation of Fo (STD). (C) Phonatory fundamental frequency range (PFR). (D) Jitter ratio. (E) Noiseto-harmonic ratio (NHR). PREO = preoperative evaluation; EPOE = early postoperative evaluation; IPOE = intermediate postoperative evaluation; $\mathrm{LPOE}=$ late postoperative evaluation; LTPOE = long-term postoperative evaluation; VLTPOE $=$ very long-term postoperative evaluation.
In the field of laryngology, previous animal studies investigated the fate of modified HA injected in the VFs of an animal model: dextranomeres in hyaluronic (DiHa) and hylan B gel, a crossed-linked HA (Hylaform, Biomatrix Inc., Ridgefield, NJ, injected in rabbit VFs were shown to be nontoxic and free of allergic as well as foreign body reactions..$^{14,15}$ Hylan B gel, largely used in the treatment of wrinkles, did not induce any inflammatory reaction when injected in the rabbit VF. Those HA derivatives have then been used in augmentative surgery as injectable materials in patients suffering of glottic insufficiency, with very good results on videostroboscopic parameters. ${ }^{16,17}$ In the case of hylan B gel, the authors noted a few patients with early signs of inflammation (within a week after injection), but all cases resolved without sequelae within 30 days.

The biopolymer used in this study (MeroGel) and the Hylan B gel (Hylaform) are both obtained from rooster combs, but modification of the molecule of HA is different: esterification in the first case and cross-linking in the second case. Hylaform presents as an injectable gel, whereas MeroGel is a solid, fiber material. The site of bioimplantation in the present study is the microdissected SLLP and not the muscle vocalis or the vocal ligament such as in previous clinical studies. It is the first time that exogenous HA has been placed extremely superficially in the human VF, immediately under the mucosal flap, and in this aspect, the fiber presentation of the EHA implant is important because it allows us to arrange it precisely before closure of the mucosal flap. The goal is also very different: we plan on a temporary action and not on a long-term residence of the HA implant in the treated VF, explaining our choice for a resorbable implant, whereas previous studies are planning on persistence of implanted modified HA.

One question raised by these clinical observations is of course the estimated time of residence of the EHA implant in the Reinke's space. According to the manufacturer of MeroGel, the implant dissolves in 2 weeks in the nose and in 6 weeks in the ear. From the observation of the laryngeal image, we interpret the disappearance of a slight convexity of the treated VF observed in three of our 
cases as the probable resorption of the inserted EHA implant: this would mean that 3 to 4 weeks are necessary for the resorption of the implant. The estimated time of residence of EHA used in this study is far enough for the goal pursued: creation of a temporary physical barrier between epithelial basement membrane and the VF ligament and creation of optimal conditions for the healing process inside the SLLP. Several recent animal studies show that levels of HA are decreased in injured VF in the early days after injury. ${ }^{18,19}$ The authors believe that low HA level produces a less than optimal environment for normal tissue regeneration and may contribute to formation of scar tissue. They state that those studies could provide a rationale reason to increase $\mathrm{HA}$ in acute VF scar to improve postoperative vocal outcomes.

\section{Perceptual Voice Evaluation Shows Good Voice Recovery after Microsurgery and Bioimplantation}

Laryngeal pathologies are usually associated with an elevation of the value of Jitt, STD, PFR, and NHR. Vocal improvement is therefore linked to a postoperative lowering of their value. Lowering of STD, PFR, and NHR shows objective vocal evolution that can be interpreted as vocal improvement in 9 cases of 11 for STD, PFR, and NHR. Jitt values exhibit less favorable vocal evolution in our series.

From the results obtained in our series of 11 consecutive EHA bio-implants, we can conclude that the EHA implant is safe and well tolerated in the micro dissected SLLP. We observed good to excellent pliability of the VF cover after treatment, especially in very bad cases such as huge organized hematoma, deep intracordal cysts, and extremely deep vergeture. Those results are encouraging and could be linked to a temporary raise of the level of HA thanks to the insertion of exogenous modified HA, which eventually favors an optimal healing and reconstitution of a new SLLP in the treated VF. Of course, the small size of this series and the absence of control cases forbid us to conclude to an improvement of SLLP's pliability because of the use of such an implant. Nevertheless, the use of such an implant easy to arrange under the mucosal flap, because of its fiber structure, could be an important help in the treatment or the prevention of cordal scars. The implant would cushion the dissected scarred mucosa and maybe favor regeneration of a thicker and more pliable SLLP. Previous studies evaluating viscoelastic shear properties of various implantable biomaterials already stressed that biomechanically speaking, HA could be a potentially optimal bio-implant for the surgical management of VF mucosa defects and LP deficiencies. ${ }^{17,20,21}$

\section{CONCLUSION}

In this pilot study, the use of exogenous modified HA in the microdissected SLLP of 11 consecutive cases treated for benign VF lesions showed no adverse reaction during the time of follow-up (between 8 weeks and 19 months). Excellent pliability of the mucosal cover was obtained postoperatively, particularly in cases where mucosal stiffness was extremely important preoperatively.
These good results obtained in these first clinical experiences have to be confirmed by larger series and long-term follow-up in the latest treated cases. Controlled studies are necessary in the future to evaluate the role of implantation of modified HA in microdissected SLLP on the phonatory and mucosal pliability outcomes.

\section{BIBLIOGRAPHY}

1. Hirano M. Morphological structure of the vocal cord as a vibrator and its variations. Folia Phoniatr (Basel) 1974;26: 89-94.

2. Ward PD, Thibeault SL, Gray SD. Hyaluronic acid: its role in voice. J Voice 2002;16:303-309.

3. Meyer K, Palmer JW. The polysaccharide of the vitreous humor. J Biol Chem 1934;107:629-634.

4. Butler JE, Hammond TH, Gray SD. Gender related differences of hyaluronic acid distribution in the human vocal fold. Laryngoscope 2001;11:907-911.

5. Gray SD, Titze IR, Chan RW, Hammond T. Vocal folds proteoglycan and their influence on biomechanics. Laryngoscope 1999;109:845-854.

6. Chan RW, Gray SD, Titze IR. The importance of hyaluronic acid in vocal folds biomechanics. Otol Laryngol Head Neck Surg 2001;124:607-614.

7. Balaszea Larsen NE. Hyaluronan: aiming for perfect skin regeneration. In: Garg HG, Longaker MT, eds. Scarless Wound Healing. New York: Marcel Dekker, 2000;143-160.

8. Ford CN. Advances and refinements in phonosurgery. Laryngoscope 1999;109:1891-1900.

9. Courey MS, Garrett CG, Ossoff RH. The medial microflap for excision of benign vocal fold lesions. Laryngoscope 1997; 107:340-344.

10. Bless DM, Rousseau B, Welham N, et al. Fibronectin and adhesion molecules on canine scarred vocal folds. Laryngoscope 2003;113:966-972.

11. Benninger MS, Alessi D, Archer S, et al. Vocal fold scarring : current concepts and management. Otolaryngol Head Neck Surg 1996;115:474-482.

12. Asari A. Medical applications of hyaluronan. Glycoforum Web Site. Accessed March 7, 2000. Available at http://www. glycoforum.gr.jp/science/hyaluronan/HA12/HA12E.html.

13. Medtronic Xomed Merogel technical monograph. Medtronic Xomed Web Site. Available at: http://www.medtronic.com.

14. Hallén L, Dahlqvist Å, Laurent C. Dextranomeres in hyaluronan (DIHA): a promising substance in treating vocal cord insufficiency. Laryngoscope. 1998;108:393-397.

15. Hallén L, Johansson C, Laurent C. Cross-linked hyaluronan (hylan B Gel) : a new injectable remedy for treatment of vocal fold insufficiency-an animal study. Acta Otolaryngol (Stock) 1999;119:107-111.

16. Hallén L, Testad P, Sederholm E, et al. DiHa (dextranomers in hyaluronan) injections for the treatment of insufficient closure of the vocal folds: early clinical experiences. Laryngoscope 2001;111:1063-1067.

17. Hertegård S, Hallén L, Laurent $\mathrm{C}$, et al. Cross-linked hyaluronan used as augmentation substance for treatment of glottal insufficiency: safety aspects and vocal fold function. Laryngoscope 2002;112:2211-2219.

18. Thibeault SL, Rousseau B, Welham NV, et al. Hyaluronan levels in acute vocal fold scar. Laryngoscope 2004;114: $760-764$.

19. Rousseau B, Sohn J, Montequin DW, et al. Functional outcomes of reduced hyaluronan in acute vocal fold scar. Ann Otol Rhinol Laryngol 2004;113:767-776.

20. Chan RW, Titze IR. Viscosities of injectable biomaterials in vocal fold augmentation surgery. Laryngoscope 1998;108: $725-731$.

21. Hertegård S, Dahlqvist Å, Laurent D, et al. Viscoelastic properties of rabbit vocal folds after augmentation. Otolaryngol Head Neck Surg 2003;401-406. 